\title{
Benzo(a)pyrene Enhanced Dermatophagoides Group 1 (Der f 1)-Induced TGF $\beta 1$ Signaling Activation through the Aryl Hydrocarbon Receptor-RhoA Axis in Asthma
}

\author{
Eryi Wang ${ }^{1}$, Wei Tu${ }^{1}$, Danh $\mathrm{Do}^{2}$, Shehar Bhatti ${ }^{2}$, Liteng Yang ${ }^{1}$, Xizhou Sun ${ }^{1}$, Damo Xu ${ }^{1}$, \\ Pingchang Yang ${ }^{1}$, Shau-Ku Huang ${ }^{3}$, Pei-Song $\mathrm{Gao}^{2}$, and Zhigang Liu ${ }^{1}$ \\ ${ }^{1}$ Shenzhen University \\ ${ }^{2}$ Johns Hopkins University \\ ${ }^{3}$ National Health Research Institutes, Miaoli, Taiwan
}

November 24, 2020

\begin{abstract}
Background: We have previously demonstrated that benzo(a)pyrene (BaP) co-exposure with dermatophagoides group 1 allergen (Der f 1) can potentiate Der f 1-induced airway inflammation. We sought to investigate the molecular mechanisms underlying the potentiation of BaP exposure on Der f 1-induced airway inflammation. Methods: BaP co-exposure with Der f 1-induced activation of TGF $\beta 1$ signaling was analyzed in airway epithelial cells (HBECs) and in asthma mouse model. The role of aryl hydrocarbon receptor (AhR) and RhoA in BaP co-exposure-induced TGF $\beta 1$ signaling was investigated. AhR binding sites in RhoA were predicted and experimentally confirmed by luciferase reporter assays. The role of RhoA in BaP co-exposureinduced airway hyper-responsiveness (AHR) and allergic inflammation was examined. Results: BaP co-exposure potentiates Der $\mathrm{f}$ 1-induced TGFß1 signaling activation in HBECs and in the airways of asthma mouse model. The BaP co-exposureinduced the activation of TGF $\beta 1$ signaling was attenuated by either AhR antagonist CH223191 or AhR knockdown in HBECs. Furthermore, AhR knockdown led to the reduction of BaP co-exposure-induced active RhoA. Inhibition of RhoA signaling with fasudil, a RhoA/ROCK inhibitor, suppressed BaP co-exposure-induced TGF $\beta 1$ signaling activation. This was further confirmed in HBECs expressing constitutively active RhoA (RhoA-L63) or dominant negative RhoA (RhoA-N19). Luciferase reporter assays showed prominently increased promoter activities for the AhR binding sites in the promoter region of RhoA. Inhibition of RhoA suppressed co-exposure-induced AHR, Th2-associated airway inflammation and TGF $\beta 1$ signaling activation in asthma. Conclusions: Our studies identified a functional axis of AhR-RhoA that regulates TGF $\beta 1$ signaling activation, leading to allergic airway inflammation and asthma.
\end{abstract}

\section{Hosted file}

Eryi et al-Allergy-11-23-2020 combined.pdf available at https://authorea.com/users/378417/ articles/494926-benzo-a-pyrene-enhanced-dermatophagoides-group-1-der-f-1-induced-tgf $\%$ CE\% B21-signaling-activation-through-the-aryl-hydrocarbon-receptor-rhoa-axis-in-asthma 